\title{
Restricción del crecimiento fetal e insulinorresistencia. Nuevos hallazgos y revisión de la literatura
}

\author{
Néstor Soto I ${ }^{1}$ y Verónica Mericq $\mathbf{G}^{2}$. \\ Fetal growth restriction and insulin \\ resistance. New findings and review \\ of the literature
}

\begin{abstract}
There is a strong association between low birth weight and insulin resistance. The thrifty phenotype hypothesis, which postulates fetal programming for adaptation to an adverse intrauterine environment, resulting in a lower insulin sensitivity in utero, is one of the hypothesis to explain this association. Later in life, syndrome $\mathrm{X}$ may develop, featuring hypertension, dyslipidemia, central obesity and type 2 diabetes, associated to an excessive food intake. Our investigation during the first three years of life in a prospective cohort of small (SGA) or appropriate for gestational age newborns, demonstrated that a significant increase of insulin levels is detected in SGA, as early as during the first year of life, but only when catch up growth (CUG) occurs. Orexigenic peptides such as Ghrelin appear to participate in this CUG phenomenon. We also sought to determine whether these associations were observed in individuals born with very low birth weight. We found that in utero as well as postnatal growth rates were independent determinants of insulin sensitivity and secretion. Education about feeding practices and physical activity in SGA children, is a future challenge to prevent the onset of syndrome X in this predisposed population (Rev Méd Chile 2005; 133: 97-104).
\end{abstract}

(Key-w ords: Fetal growth retardation; Insulin resistance; Metabolic syndrome X)

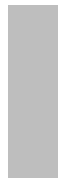

Recibido el 1 de abril, 2004. Aceptado en versión corregida el 24 de julio, 2004.

${ }^{1}$ Sección de Endocrinología y Diabetes, Hospital Clínico San Borja-Arriarán.

2Instituto de Investigaciones Materno Infantil, Facultad de Medicina Universidad de Chile y Hospital Clínico San Borja-Arriarán. Santiago de Chile.

Trabajo financiado por Proyecto Fondecyt \# 1000939.

$\mathrm{U}$ no de los tópicos más novedosos en Endocrinología, en los últimos años, ha sido la inesperada relación descrita en 1989 por David Barker entre el bajo peso de nacimiento (BPN) y las alteraciones metabólicas asociadas a mayor riesgo cardiovascular en la edad adulta ${ }^{1}$. Los estudios epidemiológicos retrospectivos originales,

Correspondencia a: Dr. Néstor Soto. Santa Rosa 1234, $6^{\circ}$ piso, Servicio de Medicina Interna. Fono: 5446600 Fax: 5546602. E-mail: nestsoto@mi.cl fueron realizados en sujetos en la sexta década de vida y demostraron que las personas con BPN tendrían mayor predisposición a desarrollar hipertensión arterial ${ }^{2}$, diabetes mellitus tipo $2^{3}$, dislipidemia y obesidad central, configurando el llamado síndrome metabólico o síndrome $\mathrm{X}^{4}$. Múltiples estudios epidemiológicos posteriores han confirmado esta relación en distintos grupos étnicos y en edades más tempranas, con resultados generalmente concordantes con la hipótesis ${ }^{5-8}$. 
En la patogenia de la diabetes tipo 2, clásicamente, se considera la coexistencia de una alteración simultánea en la sensibilidad y secreción de insulina ${ }^{9}$. La insulinorresistencia (IR) se ha atribuido a factores genéticos asociados a factores ambientales que la acentúan, especialmente el desarrollo de obesidad. Una de las ideas más atractivas, a partir de los estudios del grupo de Barker, es el concepto de «enotipo ahorrativo» ${ }^{0}$. Este concepto se refiere a los mecanismos de adaptación que realizan los sujetos durante la vida intrauterina, en un período crítico del desarrollo, en que para mantener la sobrevida frente a condiciones nutricionales adversas, o noxas de otro tipo, disminuye la sensibilidad a insulina. Una vez fuera del ambiente materno, si las condiciones cambian y existe una sobreoferta alimentaria, los portadores de este fenotipo ahorrativo, independiente de la causa que determinó el retraso de crecimiento fetal, estarían en mayor riesgo de desarrollar el síndrome $\mathrm{X}$ que los nacidos con peso adecuado a su edad gestacional (AEG).

Uno de los hechos epidemiológicos más relevantes, en relación al explosivo incremento de diabetes tipo 2 a nivel mundial, es que ha afectado proporcionalmente en forma mucho más marcada a países en vías de desarrollo, en los cuales la prevalencia de recién nacidos pequeños para la edad gestacional (PEG) es mayor que en los países desarrollados ${ }^{11,12}$. El cambio en el estilo de vida en los últimos años a uno más «occidental», puede ser el factor que explique la explosión de diabetes tipo 2 en dichas regiones, con una población especialmente predispuesta ${ }^{13}$.

Una de las principales limitaciones que tienen la mayoría de los estudios relacionados a esta hipótesis es su carácter retrospectivo, en los cuales se describe la situación de una población respecto a su perfil metabólico y se relaciona con el peso de nacimiento, pero se desconocía la evolución respecto a otros parámetros antropométricos y metabólicos durante los primeros años de vida. Si la hipótesis fuera correcta, era esperable que el fenómeno se evidenciara precozmente en la vida. Este fue el objetivo que tuvo nuestro grupo al evaluar el impacto del retardo de crecimiento intrauterino en la sensibilidad a insulina en los primeros tres años de vida, para lo cual iniciamos un estudio de cohorte de un grupo de recién nacidos de término PEG (peso de nacimiento menor que percentil 5) versus un grupo AEG (peso de nacimiento mayor que percentil 10) en una población de recién nacidos de estrato socioeconómico similar, medio bajo, sin diferencias en la talla de sus padres, evaluando parámetros antropométricos y metabólicos en las primeras 48 horas, al año, dos y tres años de vida.

A las 48 horas de vida, los recién nacidos PEG presentan niveles significativamente más bajos de glicemia e insulinemia y significativamente más altos de IGFBP-1, SHBG, ß-hidroxibutirato y ácidos grasos libres, dando cuenta de un mayor efecto de insulina sobre el control de glicemia pero menor sobre el resto de las acciones de insulina en esa etapa ${ }^{14}$. Cuando se evalúa lo que ocurre al año de vida este fenómeno se revierte, destacando una clara tendencia a elevación de los triglicéridos en el grupo PEG, sin diferencias significativas en glicemia e insulinemia entre PEG y $\mathrm{AEG}^{15}$.

En 1999, Erikson analizó el efecto del crecimiento compensatorio (CC) en los recién nacidos PEG en relación al riesgo de muerte por enfermedad coronaria y concluyó que las mayores tasas de muerte ocurren en sujetos nacidos PEG que tuvieron un peso normal o sobre el promedio a la edad de 7 años $^{16}$. Este y otros antecedentes, como el estudio de Ong $^{17}$, que relaciona el CC postnatal con el desarrollo de obesidad, nos instaron a analizar nuestra cohorte, separando a los PEG según hubieran presentado o no $\mathrm{CC}$ y se evidencia que, ya al año de edad, el grupo de PEG con CC en peso tiene un nivel de insulina en ayunas significativamente mayor que el grupo PEG sin CC y que el grupo AEG, pese a un menor índice de masa corporal. Al separar los grupos por CC en talla, el grupo PEG con CC tiene niveles de insulina significativamente más elevados, tanto basales como durante la estimulación con glucosa intravenosa. Esto nos llevó a concluir que la sensibilidad a insulina estática está relacionada al CC en peso y que la secreción insulínica estaría más determinada por el CC en talla ${ }^{15}$. Probablemente el crecimiento acelerado en los niños PEG durante este período de la vida podría ocurrir en forma desproporcionada, condicionando una composición metabólicamente desfavorable. 
Nuestros resultados son concordantes con estudios previos que demuestran, en edades más tardías, que el hecho de nacer de bajo peso y presentar CC se asocia a un mayor riesgo de síndrome $\mathrm{X}^{18}$.

No todos los estudios confirman la hipótesis que relaciona el bajo peso de nacimiento con enfermedades del adulto. En un estudio casocontrol realizado en 132 parejas de gemelos discordantes para infarto de miocardio, Hubinette no encontró diferencias en los parámetros antropométricos al nacimiento comparando los gemelos sanos con los que habían presentado infarto de miocardio ${ }^{19}$. Otros autores como Poulter ${ }^{20}$ y Kramer $^{21}$ también plantean que la asociación no sería relevante. En el Early Bird Study, que incluyó 300 niños, la IR a los 5 años no estuvo relacionada al peso de nacimiento, pero sí con el peso actual y CC en peso en ambos $\operatorname{sexos}^{22}$. Por otra parte, Dwyer analizó la antropometría al nacimiento y a los 8 años en 298 niños, además de mediciones de insulina, glucosa, lípidos y presión arterial y encontró que la presión arterial se correlacionó con adiposidad y peso al nacimiento y la masa grasa actual se correlacionó con IR, pero ni la IR ni la dislipidemia estuvo asociada con la antropometría al nacimiento ${ }^{23}$.

El mecanismo que relaciona el bajo peso de nacimiento con la hipertensión arterial no está bien definido. Se ha atribuido al exceso de insulina un rol patogénico directo. También a una alteración del eje hipotálamo-hipófisis-adrenal y actividad adrenomedular. Se ha evaluado la respuesta de cortisol en una población de adultos jóvenes no obesos ${ }^{24}$, encontrándose que tanto el cortisol basal como en respuesta a ACTH está elevado en los nacidos PEG, presentando una mayor presión arterial sistólica y diastólica, pese a mantener menor peso y talla que el grupo control AEG. Por otra parte, Tenhola ${ }^{25}$ demostró que los nacidos PEG tenían niveles de DHEA-S y epinefrina, pero no de norepinefrina, más elevados que su contraparte de igual edad nacidos AEG, aunque no evaluaron cifras de presión arterial. Recientemente, Boguszewski describió, midiendo directamente, una mayor actividad simpática en el lecho vascular del tejido muscular ${ }^{26}$. También se demostró alteraciones del eje suprarrenal en los nacidos PEG en estudios de Francois ${ }^{27}$ e Ibáñez ${ }^{28}$, en que niveles elevados de DHEA-S se acompañan de adrenarquia precoz. Otra explicación al aumento del cortisol postnatal en niños PEG surge de experimentos realizados por el grupo de Seckl ${ }^{29}$, en modelos animales sujetos a desnutrición in utero, en los cuales ha documentado una disminución de la actividad de la enzima 11-ßdehidrogenasa tipo 2 placentaria, lo que conduce a un aumento del cortisol circulante fetal, lo cual llevaría a una disminución en la síntesis de factores de crecimiento fetales y una reprogramación de las respuestas vasculares y actividad del eje hipotálamo-hipófisis-suprarrenal, incluso a un cambio en el set point de los receptores de glucocorticoides cerebrales ${ }^{30}$. En nuestra cohorte, no encontramos diferencias en los niveles de cortisol entre niños nacidos PEG y AEG, al menos durante los primeros tres años de la vida.

La dislipidemia es un elemento característico del síndrome X. En nuestro estudio destacó el hallazgo de niveles de triglicéridos significativamente más altos en el grupo PEG al año de vida, pero no hubo diferencias respecto a colesterol en esta fase de la vida, como se ha demostrado a edades más tardías ${ }^{31}$. Un estudio previo, realizado en nuestro país ${ }^{32}$, comparó los niveles de lípidos, medidos en sangre venosa del cordón en recién nacidos bajo el percentil 10 con un grupo normal, encontrando una elevación en los niveles de triglicéridos en nacidos con retraso de crecimiento asimétrico, pero sin diferencias en colesterol total, HDL, LDL o apoproteínas A1 y B. Tenhola evaluó la concentración de lípidos séricos a la edad de 12 años y observó que cerca de la mitad de los nacidos PEG estaban en el cuartil mayor para colesterol total del grupo control AEG y que los PEG con mal CC en talla pueden tener mayor riesgo de hipercolesterolemia, sin embargo no hubo diferencias en las concentraciones de lípidos entre los dos grupos ${ }^{33}$. Estos hallazgos difieren con los de Bavdekar ${ }^{18}$ en una cohorte mayor, evaluada a los 8 años, en que tanto el HOMA-IR como el colesterol total fueron mayores a menor peso de nacimiento y mayor peso actual. Adultos jóvenes nacidos PEG que muestran hiperinsulinemia en ayunas tienden a tener hipertrigliceridemia en comparación a controles de igual edad, pero esta diferencia desaparece cuando se ajusta por el nivel de insulina ${ }^{34}$. 
Un elemento no aclarado en los estudios previos era conocer si la disminución a la sensibilidad insulínica postnatal de los niños nacidos PEG estaba solo presente cuando ocurría una condición adversa in utero o también podía presentarse ante la exposición a condiciones adversas postnatales, como es en casos de prematurez extrema. Otra interrogante era conocer el efecto que tenía la prematurez, dado que en la mayoría de los estudios el peso de nacimiento era evaluado independientemente de la edad gestacional o los niños pretérmino fueron excluidos. Recientemente se han respondido estas preguntas en un estudio de Bazáes ${ }^{35}$ en niños nacidos de muy bajo peso de nacimiento $(690-1.500 \mathrm{~g})$, con edades gestacionales entre 25-34 semanas, estudiados a la edad promedio de 5,7 años, que cuentan con un completo seguimiento antropométrico desde la edad de 2 semanas. En este grupo de niños prepuberales se apreció que los nacidos PEG presentan menor sensibilidad a insulina que los AEG, efecto que persistía al ajustar por índice de masa corporal actual, edad gestacional y morbilidad perinatal. En este estudio, la secreción de insulina en ayunas y postcarga de glucosa intravenosa se correlacionó significativamente con tasas de crecimiento postnatal temprano.

La asociación entre el crecimiento fetal reducido e IR ha sido demostrada ampliamente usando diversos métodos, sin embargo, los sujetos PEG muestran una susceptibilidad variable a IR, con un amplio rango en la captación de glucosa mediada por insulina durante el clamp euglicémico hiperinsulinémico ${ }^{36}$. Esta respuesta variable puede ser explicada por una diferente susceptibilidad genética. Varios polimorfismos de genes involucrados en el desarrollo y función metabólica del tejido adiposo y muscular modulan la susceptibilidad a IR en humanos. Jaquet ${ }^{37}$ evaluó el efecto de tres polimorfismos en IR durante una prueba de tolerancia a glucosa oral, comparando sujetos de 20 años de edad nacidos PEG o AEG. Estudió polimorfismos del factor de necrosis tumoral $\alpha$, del receptor $ß 3$ adrenérgico y del receptor activador de proliferación de peroxisomas (PPAR). Los portadores del alelo TNFa/G-308 tuvieron un nivel de insulina en ayunas y relación insulina/ glucosa significativamente mayor que los no por- tadores en los nacidos PEG y no hubo diferencias en los AEG. Un patrón de respuesta similar se observó en los portadores de los alelos PPARy 2/ Pro12A1a y ADRB 3/G+250 comparando con los no portadores de estos alelos. Para todos los parámetros hubo una interacción significativa entre el efecto genético y el tamaño al nacer. No hubo diferencia significativa entre PEG y AEG en relación a la frecuencia de alelos. Ellos concluyen que la mayoría de los genes de susceptibilidad a enfermedades multifactoriales no tienen un rol causal primario sino que actúan como modificadores de respuesta a factores ambientales como dieta, actividad física y condiciones patológicas.

Otro estudio, destinado a demostrar si la respuesta secretora de insulina frente a un estímulo puede estar determinada genéticamente es el de Bazáes $^{38}$, que analiza si existe relación entre los haplotipos de una zona del promotor del gen de insulina variable number of tandem repeats (INS VNTR), la secreción de insulina y el crecimiento postnatal temprano. Se encontró que los niveles de insulina, tanto en ayunas como el área bajo la curva durante un test de tolerancia a la glucosa intravenosa, fueron mayores en los portadores del haplotipo homocigoto clase III/III que en los I/I, con una respuesta intermedia en los individuos heterocigotos, en tanto los niveles de glucosa fueron similares en ambos grupos. Las diferencias de genotipo en la sensibilidad y secreción de insulina fueron independientes del tamaño al nacer, velocidad de crecimiento postnatal e índice de masa corporal actual.

¿Qué determina que la mayoría de los niños presente CC y un subgrupo no lo presente? Uno de los determinantes probables es la mayor 0 menor disponibilidad de alimentos, pero otra explicación posible es que la ingesta alimentaria sea diferente. En la regulación de la ingesta alimentaria, uno de los péptidos estudiados en los últimos años es Ghrelina, péptido de 28 aminoácidos, producido principalmente en el fondo gástri$\mathrm{co}$, que inicialmente se conoció como estimulador de la secreción de hormona de crecimiento $(\mathrm{GH})$ y posteriormente se descubrió su importante rol como regulador rápido del apetito. Se ha demostrado que posee un efecto orexígeno que es independiente de la secreción de GH. En condiciones de ayuno y de hipoglicemia se produce un 
aumento de Ghrelina. Para evaluar el rol de Ghrelina en el CC en peso en los lactantes PEG y AEG, nuestro grupo estudió la respuesta de Ghrelina frente al estímulo de glucosa intraveno$\mathrm{sa}^{39}$. Se pudo apreciar que, al igual que lo descrito en adultos, la administración de glucosa intravenosa produce una disminución de los niveles plasmáticos de Ghrelina. En los nacidos PEG que presentaron CC, la frenación de Ghrelina frente a una carga de glucosa fue significativamente menor que en los PEG sin CC, lo que permite plantear que esta respuesta de Ghrelina se traduce en una mayor ingesta alimentaria en los niños que compensan el déficit de crecimiento durante el primer año.

Los mecanismos subyacentes para el desarrollo de IR en los PEG con crecimiento compensatorio permanecen desconocidos. Tanto la oxidación de glucosa como el depósito no oxidativo se ven afectados. Veening demostró ${ }^{40}$, en un grupo de niños de 8 años de edad, que la captación de glucosa mediada por insulina está reducida en los nacidos PEG durante un clamp euglicémico hiperinsulinémico. Se ha visto que sujetos nacidos PEG poseen una regulación alterada de la expresión de GLUT 4 mARN tanto en tejido muscular como adiposo $^{41}$, lo que refuerza el rol del transporte de glucosa en el control de la sensibilidad a insulina, pero este hallazgo también está presente en otras condiciones de insulinorresistencia.

En años recientes, se han descubierto una serie de péptidos del tejido adiposo, denominados genéricamente adipocitoquinas o adipoquinas ${ }^{42}$, que participan en la regulación de la acción insulínica. Una de estas moléculas es leptina y más recientemente descubierta, la adiponectina, que a diferencia de otras adipocitoquinas, su mARN está disminuido en el tejido adiposo de obesos y diabéticos, pero es restaurado a lo normal después de la pérdida de peso. En estudios transversales, se correlaciona inversamente con obesidad, dislipidemia diabética, enfermedad cardiovascular e IR. Se le atribuye un efecto potenciador de la acción insulínica, pero el mecanismo no ha sido aclarado. En modelos animales, se ha descrito una disminución de ácidos grasos libres circulantes por aumento de su oxidación en el músculo esquelético, sumado a una disminución de ácidos grasos libres en el hígado. También activa directamente la captación de glucosa en adipocitos y músculo, actuando sobre la AMPproteinquinasa. Por lo tanto, a mayor nivel de adiponectina existirá mejor acción de insulina y en consecuencia un nivel de insulinemia menor. Nuestro grupo determinó el nivel de adiponectina en recién nacidos PEG versus AEG durante los primeros dos años de vida, no encontrándose diferencias a esa edad. Un hallazgo notable de este estudio es que, fisiológicamente, se produce una caída significativa del nivel de adiponectina entre el primer y segundo año de vida y que la respuesta difiere por sexo, con un menor nivel en mujeres a los dos años. Encontramos una correlación inversa entre las concentraciones de adiponectina y la diferencia de peso entre el primer y segundo año en los PEG pero no en los $\mathrm{AGA}^{43}$. Un hallazgo distinto al nuestro ha sido descrito recientemente por Cianfarani en niños de 8,6 años, en que los niveles de adiponectina fueron significativamente menores en los nacidos $\mathrm{PEG}^{44}$.

En conclusión, se puede afirmar, a la luz del conocimiento actual, que la hipótesis de Barker que relaciona el bajo peso de nacimiento con la IR es correcta. Los sujetos con un genotipo predispuesto tendrían mayor posibilidad de desarrollar una disminución de sensibilidad a insulina, la que afecta preferentemente a los nacidos PEG que presentan CC y es posible observar este fenómeno tan precozmente como al año de vida. La implicancia clínica práctica que tienen estos hallazgos está dada por la necesidad de identificar y prevenir en etapas precoces la aparición del fenómeno de IR, el cual es cada vez más frecuente en nuestra sociedad actual.

La prevención de la obesidad en la infancia puede ser aún más beneficiosa en niños que nacen PEG, pero idealmente, el enfoque preventivo futuro debería orientarse a prevenir el retardo de crecimiento intrauterino. 


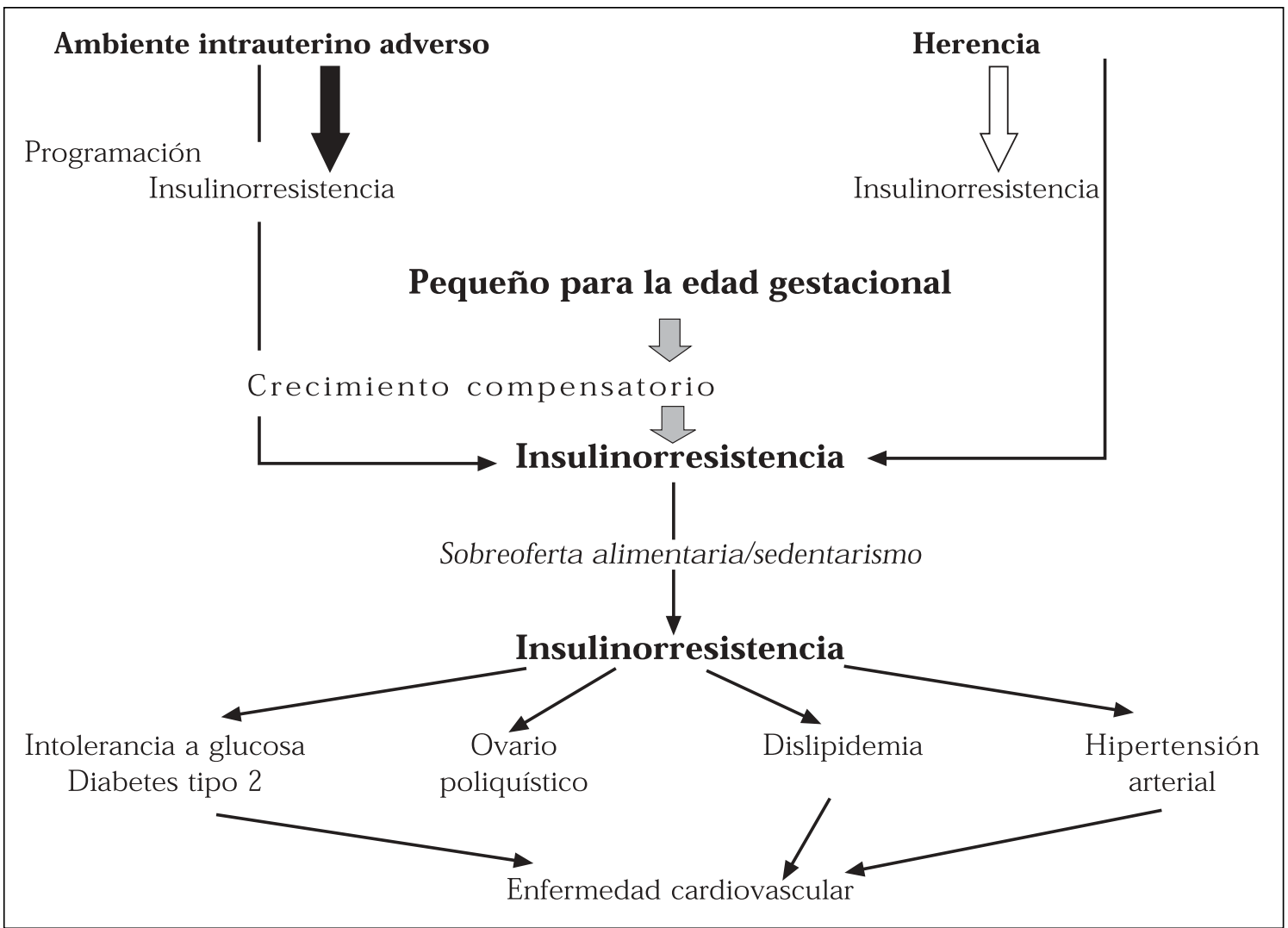

Figura 1. Esquema que explica la hipótesis de la asociación entre la restricción de crecimiento fetal e insulinorresistencia.

\section{REFERENCIAS}

1. Barker DJP, Osmond C, Golding J, Kuh D, WADSWORTH ME. Growth in utero, blood pressure in chilhood and adult life, and mortality from cardiovascular disease. BMJ 1989; 298: 564-7.

2. Barker DJP, Ball AR, Osmond C, Simonds SJ. Fetal and placental size and risk of hypertension in adult life. BMJ 1990; 301(6746): 259-62.

3. Hales CN, Barker DJP, Clark PM, Cox LJ, Fall C, Osmond C, Winter PD. Fetal and infant growth and impaired glucose tolerance at age 64. BMJ 1991; 303: 1019-22.

4. Barker DJP, Hales CN, Fall CHD, O smond C, Phipps $\mathrm{K}$, Clark PM. Type 2 (non-insulin dependent) diabetes mellitus, hypertension and hyperlipidemia (syndrome $\mathrm{X}$ ): relation to reduced fetal growth. Diabetologia 1993; 36: 62-7.
5. Bo S, Cavalo-Perin P, Scaglone L, Ciccone G, Pagano G. Low birthweight and metabolic abnormalities in twins with increased susceptibility to Type 2 diabetes mellitus. Diabet Med 2000; 17: 365-70.

6. Poulsen P, Vaag AA, Kyvik KO, Moler Jensen D, BECK-NieLSEN H. Low birth weight is associated with NIDDM in discordant monozygotic and dizygotic twin pairs. Diabetologia 1997; 40: 43946.

7. Ravew AC, Van Der Meulen JH, Michels RP, O smond C, Barker DJ, Hales CN, Bleker OP. Glucose tolerance in adults after prenatal exposure to famine. Lancet 1998; 351: 173-7.

8. Valdez R, Athens MA, Thompson GH, Bradshaw BS, STERN MP. Birthweight and adult health outcomes in a biethnic population in the USA. Diabetologia 1994; 37: 624-31. 
9. FERRANNINI E. Insulin resistance versus insulin deficiency in non-insulin dependent diabetes mellitus: problems and prospects. Endocr Rev 1998; 19(4): 477-90.

10. Hales CN, Barker DJP. Type 2 (non insulin dependent) diabetes: the thrifty phenotype hypothesis. Diabetologia 1992; 35: 595-601.

11. Y АJNIK CS. Early life origins of insulin resistance and type 2 diabetes in India and other Asian countries. J Nutr 2004 Jan; 134(1): 205-10.

12. Yajnik CS, Fall CH, Coyaji KJ, Hirve SS, Rao S, BARKER DJ ET AL. Neonatal anthropometry: the thin-fat Indian baby. The Pune Maternal Nutrition Study. Int J Obes Relat Metab Disord 2003 Feb; 27(2): 173-80.

13. King H, Aubert RE, HeRman WH. Global burden of diabetes, 1995-2025: prevalence, numerical estimates and projections. Diabetes Care 1998: 1414-31.

14. Bazáes Ra, Salazar Te, Pittaluga E, Peña V, Alegría A, IÑíguez $G$ et AL. Small for gestational age infants at 48 hours of age. Pediatrics 2003; 111 (4Pt 1): 804-9.

15. Soto N, Bazáes RA, Peña V, Salazar T, Avila A, IÑ́GGUEZ $G$ ET AL. Insulin sensitivity and secretion are related to catch up growth in small-forgestational age infants at age 1 year: results from a prospective cohort. J Clin Endocrinol Metab 2003; 88: 3645-50.

16. Erikson JG, Forsen T, Tuomilehto J, Winter PD, OSMOND C, BARKER DJP. Catch up growth in childhood and death from coronary heart disease: longitudinal study. BMJ 1999; 318: 427-431.

17. Ong KK, Ahmed ML, Emmet PM, Preece MA, Dunger DB. Association between postnatal catch-up growth and obesity in childhood: prospective cohort study. BMJ 2000; 320: 967-71.

18. Bavdekar A, Yaknik CS, Fall CH, Bapet S, Pandit AN, Deshpade V et al. Insulin resistance syndrome in 8-year-old Indian children: small at birth, big at 8 years, or both? Diabetes 1999; 48: 2422-9.

19. Hubinette A, Cnattingius S, Ekborm A, De Faire U, KRAMER M, LICHTENSTEIN ET AL. Birthweight, early environment, and genetics: a study of twins discordant for acute myocardial infarction. Lancet 2001; 357: 1997-2001.

20. PoulteR NR. Birthweights, maternal cardiovascular events and Barker hypothesis. Lancet 2001; 357: 1990-91.

21. KRAMER MS. Invited commentary: association between restricted fetal growth and adult chronic disease: is it causal? Is it important? Am J Epidemiol 2000; 152: 605-8.

22. WILKIN TJ. The relative contribution of birth weight, weight change and current weight to insulin resistance in contemporary 5 years old: The Early Bird Study. Diabetes 2002; 51: 3468-72.

23. Dwyer T, Blizzard L, Venn A, Stankovich JM, Ponsonby AL, Morley R. Syndrome X in 8-y-old Australian children: stronger associations with current body fatness than with infant size or growth. Int J Obes Relat Metab Disord 2002; 26: 1301-9.

24. Levitt N, Lambert E, Woods D, Haies N, Andrew R, Seckl J. Impaired Glucose Tolerance and Elevated Blood Pressure in Low Birth Weight, Nonobese, Young South African Adults: Early Programming of Cortisol Axis. J Clin Endocrinol Metab 2000; 85: 4611-8.

25. Tenhola S, Martikainen A, Rahiala E, Parviainen M, HaLonen P, Voutilainen R. Increased adrenocortical and adrenomedullary hormonal activity in 12year-old children born small for gestational age. J Pediatr 2002; 141: 477-82.

26. Boguszewski MC, Johannsson G, Fortes LC, SverRIsротті YB. Low birth size and final height predict high sympathetic nerve activity in adulthood. J Hypertens 2004; 22: 1157-63.

27. Francois I, De Zegher F. Adrenarche and fetal growth. Pediatr Res 1997; 41: 440-2.

28. Ibáñez L, Potau N, Marcos MV, De Zegher F. Exaggerated adrenarche and hyperinsulinism in adolescent girl born small for gestational age. J Clin Endocrinol Metab 1999; 84: 4739-41.

29. SECKL JR. Physiologic programming of the fetus. Clin Perinatol 1998; 25: 939-62.

30. Weaver IC, Diorio J, Seckl JR, Szyf M, Meaney MJ. Early environmental regulation of hippocampal glucocorticoid receptor gene expression: characterization of intracellular mediators and potential genomic target sites. Ann N Y Acad Sci 2004; 1024: 182-212.

31. Barker DJP, Martyn CN, Osmond C, Hales CN, Fall CHD. Growth in utero and serum cholesterol concentrations in adult life. BMJ 1993; 307: 15247.

32. Molna M, Casanueva E, Cid X, Ferrada MC, Pérez R, Dios G et aL. Perfil lipídico en recién nacidos con retardo del crecimiento intrauterino. Rev Méd Chile 2000; 128: 741-8.

33. Tenhola S, Martikainen A, Rahiala E, Herrgard E, Halonen P, Voutilainen R. Serum lipid concentra- 
tions and growth characteristics in 12-year-old Children Born Small for Gestational Age. Pediatr Res 2000; 48: 623-28.

34. Jaquet D, Leger J, Levy-Marchal C, Czernichow P. Low Birth Weight: Effect on Insulin Sensitivity and Lipid Metabolism. Horm Res 2003; 59: 1-6.

35. Bazáes RA, Alegría A, Pittaluga E, Avila A, IÑíguez G, MERICQ V. Determinants of Insulin Sensitivity and Secretion in Very-Low-Birth-Weight Children. J Clin Endocrinol Metab 2004; 89: 1267-72.

36. Jaquet D, Gaboriau A, Czernichow P, Levy-Marchal C. Insulin resistance early in adulthood in subjects born with intra-uterine growth retardation. J Clin Endocrinol Metab 2000; 85:1401-6.

37. Jaquet D, Tregouet D, Godefroy T, Nicaud V, Chevenne D, Tiret L et al. Combined Effects of Genetic and Environmental Factors on Insulin Resistance Associated With Reduced Fetal Growth. Diabetes 2002; 51: 3473-8.

38. Bazáes RA, Petry CJ, Ong KK, Avila A, Dunger DB, MERICQ MV. Insulin gene VNTR genotype is associated with insulin sensitivity and secretion in infancy. Clin Endocrinol (Oxf) 2003; 59: 599-603.

39. Iníguez G, Ong K, Peña V, Avila A, Dunger D, MERICQ MV. Fasting and postglucose ghrelin levels in small for gestational age infants, relation with size and weight gain at one year of age. J Clin Endocrinol Metab 2002; 87: 5830-3.

40. Veening MA, Van Weissenbruch MM, Dejamarre-Van De WAAL HA. Glucose tolerance, insulin sensitivity and insulin secretion in children born small for gestational age. J Clin Endocrinol Metab 2002; 87: 4657-61.

41. Jaquet D, Vidal H, Hankard R, Czernichow P, LevyMARCHAL C. Impaired regulation of glucose transporter in gene expresión in insulin resistance associated with in utero undernutrition. J Clin Endocrinol Metab 2001; 86: 3266-71.

42. Pittas AG, Joseph NA, GreensBerg AS. Adypocytokynes and Insulin Resistance. J Clin Endocrinol Metab 2004; 89: 447-52.

43. Iñiguez $G$, Soto $N$, Avila A, Salazar $T$, Ong $K$, Dunger D, Merice MV. Adiponectin levels in the first two years of life in a prospective cohort. Relation with weight gain, leptin levels and insulin sensitivity. J Clin Endocrinol Met 2004; 89: 5500-3.

44. Cianfarani S, Martínez C, Maiorana A, Scire G, Spadoni GL, Boem S. Adiponectin levels are reduced in children born small for gestational age and are inversely related to postnatal catch-up growth. J Clin Endocrinol Metab 2004; 89: 1346-51. 